\title{
Changes in five-year relative survival rates in Poland in patients diagnosed in the years 1999-2010
}

\author{
Urszula Wojciechowska, Joanna Didkowska
}

Introduction. Survival rates are a tool that allows evaluation of healthcare quality and cancer treatment efficiency. The main aim of this article is to present tendencies in curability of Polish patients diagnosed between 1999-2010 and followed up until the end of 2015.

Materials and methods. Survival analysis was performed using data from the Polish National Cancer Registry and Statistics Poland. The survival rates were calculated using Hakulinen method.

Results. In Poland, five-year survival rates in men for all cancers increased from 32.9\% (diagnosis 1999-2001) to 41.3\% (diagnosis 2008-2010), whereas in women, from 50.9\% to 56.1\% respectively. The biggest improvement was found in therapy of prostate cancer (by 15.5 percentage points), hematological malignancies (7-13 p.p.), malignancies of bone and cartilage (10-14 p.p.), thyroid gland cancers (5-9 p.p.) and kidney cancer (7-9 p.p.).

Discussion. Although survival rates are lower than in other European countries, survival of oncology patients in Poland has improved.

\section{Conclusions}

1. In Poland, survival of patients with majority of cancer types has improved.

2. The greatest increase in survival rates was observed for rare malignancies.

3. The improvement occurred during the time when the National Cancer Control Program was implemented.

NOWOTWORY J Oncol 2017; 67, 6: 349-358

Key words: cancer, cancer registry, relative survival, treatment efficiency

\section{Introduction}

In Poland, malignant neoplasms have become a still growing burden to the society on many levels, however, the most noticeable are health effects. Also in other (e.g. social, organizational, and financial) dimensions, cancers pose a challenge for the healthcare system. The anticipated increase in the number of cases, resulting from aging of modern societies, including the Polish population, but also from growing exposition to some risk factors, stimulates search for strategies that would prevent cancer effects [1, 2]. These strategies are targeted mainly at popularization of primary [3] and secondary [4] prevention [3], but also at providing patients with equal accessibility of therapy [5], psychological care and rehabilitation.

Since funding of healthcare is limited, evidence-based assessment of each of these activities should be perfor- med. In European countries, where national strategies for cancer control were implemented, incidence, mortality and 5-year survival rates were deemed measures of healthcare intervention efficiency. Survival is one of the most important synthetic measures of abilities and performance of healthcare.

Survival rates are a universal measure allowing assessment of broadly understood healthcare and efficiency of treatment for particular diseases; they are used both in population studies, as well as in smaller, selected groups of patients. In clinical studies, patient survival is usually assessed according to Kaplan-Meier method, mostly to compare treatment results between specific medications or treatment regimes. These studies are usually conducted in small groups of patients and they do not reflect results obtained for a general population. Assessment of treatment 
efficiency at the level of populations from various countries provides a different perspective. In such a case, especially when no population registry is available, survival analysis is performed via an indirect method, using MIR (mortality to incidence ratio), which is a kind of a surrogate for a 5-year survival rate. This approach was used to assess healthcare functioning in the scope of oncology in OECD countries [7].

In countries where cancer registries are held, population efficiency of cancer treatment is assessed based on the so-called 5-year survival rates. A 5-year horizon was determined arbitrarily in 1930-ies, when most treatment failures occurred within 5 years of diagnosis [8]. The observed survivals do not account for the processes of natural mortality causes not related to cancer, which renders comparison in time and between populations difficult. Considering the natural mortality in the studied population allows obtaining a measure - relative survival — that is free of these limitations [9].

The last population survival rates for Poland presented in 2013 concerned only patients followed up until the end of 2010 [10]. The main aim of this article is to present long-term tendency in curability of Polish patients diagnosed between 1999-2010, for whom the treatment effects were evaluated based on the 5-year relative survival rates estimated after follow up until the end of 2015.

\section{Materials and methods}

Survival analysis was performed based on a set of cancer cases diagnosed in 1999-2010 and entered into the Polish National Cancer Registry. For over 20 years, the data in the Polish National Cancer Registry has been collected according to guidelines of the International Agency for Research on Cancer (IARC), which guarantees its comparability in time. Analysis of the set quality is performed in real time; additionally, once a year, the whole set is verified against the international guidelines. Vital status of the patients was verified based on death registry until 31 December 2015, obtained from Statistics Poland.

The cancers were coded according to the International Statistical Classification of Diseases and Related Health Problems (revision 10) valid in Poland [11]. The analysis was performed for all cancers combined and for 26 most frequent cancer localizations in adults.

The initial set covered 1,579,393 cases of cancer. Entries related to cancer cases in patients aged 0-14 were excluded from the analyzed set. Other exclusion criteria for the set included: cases registered based on autopsy or death certificate only (DCO), cases of skin cancers different than melanoma (C44) and in situ cancers (D00-D09), patients without PESEL (personal identity number) and patients for whom time interval between the date of disease onset and date of death was less than 30 days. Eventually, the analysis included 1,297,779 cases aged 15-99, diagnosed with malignant neoplasms classified in the range of C00-C96 (without skin cancer - C44) (Table I).

The estimated 5-year relative survival rates were calculated as per Hakulinen method, using SURV3 software [12]. Lifespan tables for the Polish population between 1999-2015 used in the analysis were obtained from Statistics Poland in Warsaw. This analysis compares four 3-year periods covering patients diagnosed between 1999-2001, 2002-2004, 2005-2007 and 2008-2010 and followed up until 31 December 2015.

Table I. Data quality indicators for patients diagnosed during 1999-2010

\begin{tabular}{|c|c|c|c|c|c|c|c|c|}
\hline & \multicolumn{2}{|c|}{ 1999-2001 } & \multicolumn{2}{|c|}{ 2002-2004 } & \multicolumn{2}{|c|}{ 2005-2007 } & \multicolumn{2}{|c|}{ 2008-2010 } \\
\hline \multirow[t]{3}{*}{ Number of cases in the NCR database } & \multicolumn{2}{|c|}{354,743} & \multicolumn{2}{|c|}{380,825} & \multicolumn{2}{|c|}{411,268} & \multicolumn{2}{|c|}{432,557} \\
\hline & \multicolumn{8}{|c|}{ Cases excluded from the analysis } \\
\hline & Number & $\%$ & Number & $\%$ & Number & $\%$ & Number & $\%$ \\
\hline No identity number - lost to follow-up & 5258 & $1.5 \%$ & 1189 & $0.3 \%$ & 228 & $0.1 \%$ & 115 & $0.03 \%$ \\
\hline Death within 0-29 days & 19,071 & $5.4 \%$ & 23,972 & $6.3 \%$ & 24,687 & $6.0 \%$ & 23,684 & $5.5 \%$ \\
\hline In situ cancers & 2144 & $0.6 \%$ & 3276 & $0.9 \%$ & 4717 & $1.1 \%$ & 6845 & $1.6 \%$ \\
\hline DCO & 23,756 & $6.7 \%$ & 19,481 & $5.1 \%$ & 12,227 & $3.0 \%$ & 3192 & $0.7 \%$ \\
\hline Age 0-14 & 2400 & $0.7 \%$ & 2363 & $0.6 \%$ & 2326 & $0.6 \%$ & 2278 & $0.5 \%$ \\
\hline \multirow[t]{4}{*}{ Skin cancer (C44) } & 18,388 & $0.7 \%$ & 22,609 & $0.6 \%$ & 27,432 & $0.6 \%$ & 29,454 & $0.5 \%$ \\
\hline & \multicolumn{8}{|c|}{ Cases included to the analysis } \\
\hline & \multicolumn{2}{|c|}{ 1999-2001 } & \multicolumn{2}{|c|}{ 2002-2004 } & \multicolumn{2}{|c|}{ 2005-2007 } & \multicolumn{2}{|c|}{ 2008-2010 } \\
\hline & Number & $\%$ & Number & $\%$ & Number & $\%$ & Liczba & $\%$ \\
\hline All cases & 282,490 & $79.6 \%$ & 308,106 & $80.9 \%$ & 339,954 & $82.7 \%$ & 367,229 & $84.9 \%$ \\
\hline Histopathology confirmation & 201,005 & $71.2 \%$ & 249,156 & $80.9 \%$ & 285,494 & $84.0 \%$ & 318,876 & $86.8 \%$ \\
\hline
\end{tabular}


In the following Results, "survival rates" should be understood as "5-year relative survival rates."

\section{Results}

In Poland, 5-year survival rates for patients with all cancers increased in men from $32.9 \%$ (diagnosis in 1999-2001) to $41.3 \%$ (diagnosis in 2008-2010) (increase by 8.4 percentage points); in women, the increase from $50.9 \%$ to $56.1 \%$ (increase by 5.1 p.p.), respectively, was observed.

\section{Men}

In male population (Table II, Fig. 1), the greatest survival rate increase was found for prostate cancer. Among patients diagnosed in the last analyzed period, the survival rate was 14.9 percentage points higher than in the first analyzed period and equal to $75.8 \%$.
Between 1999-2010 (follow up until the end of 2015), survival rates for Hodgkin's lymphoma patients increased by 13 p.p. (from $69.2 \%$ to $82.2 \%$ ), for patients with non-Hodgkin's lymphoma by 10.9 p.p. (from $42.8 \%$ to $53.6 \%$ ), with multiple myeloma by 9.5 p.p. (from $28.6 \%$ to $38.1 \%$ ), for leukaemia patients (C91-C95) by 7.3 p.p. (from $37.7 \%$ to $45.0 \%$ ), and for lymphatic leukaemia patients by 7 p.p. (46.8\% to $53.9 \%$ ). For myeloid leukaemia, the survival rate increased by 3 p.p. (from $25.1 \%$ to $28.1 \%$ ).

Survival rate for male patients with bone and cartilage malignancies increased by 10.0 p.p. In patients with thyroid gland cancers diagnosed between 2008-2010, higher survival rates (84.8\%) were observed as compared to patients diagnosed 8-10 years earlier: increase by 9.1 p.p. Improvement of survival was also found in patients with kidney

Table II. Five-year relative survival rates in Poland for patients at age 15-99 with diagnosis in one of four calendar period between 1999-2010

\begin{tabular}{|c|c|c|c|c|c|c|c|c|}
\hline \multirow[t]{2}{*}{ Site } & \multirow[t]{2}{*}{ ICD-10 } & \multirow{2}{*}{$\begin{array}{l}\text { Year of } \\
\text { diagnosis }\end{array}$} & \multicolumn{3}{|c|}{ Men } & \multicolumn{3}{|c|}{ Women } \\
\hline & & & Number & Rate & $95 \% \mathrm{Cl}$ & Number & Rate & $95 \% \mathrm{Cl}$ \\
\hline \multirow[t]{4}{*}{ All cancers } & \multirow[t]{4}{*}{$\mathrm{C} 00-\mathrm{C} 96$} & 1999-2001 & 142,816 & 32.9 & $32.6-33.2$ & 139,674 & 50.9 & $50.6-51.2$ \\
\hline & & 2002-2004 & 156,512 & 36.3 & $36.0-36.5$ & 151,594 & 53.1 & $52.9-53.4$ \\
\hline & & 2005-2007 & 171,933 & 39.9 & $39.7-40.2$ & 168,021 & 55.1 & $54.8-55.3$ \\
\hline & & 2008-2010 & 184,423 & 41.3 & $41.0-41.5$ & 182,806 & 56.1 & $55.8-56.3$ \\
\hline \multirow{4}{*}{$\begin{array}{l}\text { Lip, oral cavity and } \\
\text { pharynx }\end{array}$} & \multirow[t]{4}{*}{$\mathrm{C} 00-\mathrm{C} 14$} & 1999-2001 & 6439 & 36.5 & $35.2-37.7$ & 1858 & 51.3 & $48.8-53.8$ \\
\hline & & $2002-2004$ & 6720 & 35.6 & $34.3-36.8$ & 2207 & 53.5 & $51.2-55.7$ \\
\hline & & 2005-2007 & 7310 & 36.1 & $34.9-37.3$ & 2416 & 53.2 & $51.0-55.3$ \\
\hline & & 2008-2010 & 7867 & 35.8 & $34.6-36.9$ & 2876 & 52.9 & $50.9-54.9$ \\
\hline \multirow[t]{4}{*}{ Oesophagus } & \multirow[t]{4}{*}{$\mathrm{C} 15$} & 1999-2001 & 2579 & 7.2 & $6.2-8.3$ & 579 & 11.3 & $8.6-14.1$ \\
\hline & & 2002-2004 & 2672 & 6.4 & $5.4-7.4$ & 641 & 11.9 & $9.2-14.6$ \\
\hline & & 2005-2007 & 2687 & 8.2 & $7.1-9.3$ & 660 & 12.0 & $9.4-14.7$ \\
\hline & & 2008-2010 & 2682 & 7.1 & $6.1-8.2$ & 703 & 13.2 & $10.5-15.9$ \\
\hline \multirow[t]{4}{*}{ Stomach } & \multirow[t]{4}{*}{$\mathrm{C} 16$} & 1999-2001 & 8515 & 15.2 & $14.4-16.0$ & 4657 & 18.8 & $17.6-20.0$ \\
\hline & & 2002-2004 & 8698 & 15.9 & $15.1-16.7$ & 4719 & 20.3 & $19.0-21.5$ \\
\hline & & 2005-2007 & 9128 & 19.5 & $18.6-20.4$ & 5011 & 23.7 & $22.5-25.0$ \\
\hline & & 2008-2010 & 9449 & 18.9 & $18.0-19.7$ & 5117 & 24.3 & $23.1-25.6$ \\
\hline \multirow[t]{4}{*}{ Colon } & \multirow[t]{4}{*}{ C18-C19 } & 1999-2001 & 9171 & 41.8 & $40.7-42.9$ & 8971 & 44.2 & $43.0-45.3$ \\
\hline & & 2002-2004 & 11,215 & 44.9 & $43.9-45.9$ & 10,593 & 48.2 & $47.1-49.2$ \\
\hline & & 2005-2007 & 13,549 & 48.1 & $47.1-49.0$ & 12,312 & 50.8 & $49.8-51.8$ \\
\hline & & 2008-2010 & 15,230 & 48.3 & $47.4-49.2$ & 13,564 & 52.4 & $51.4-53.3$ \\
\hline \multirow[t]{4}{*}{ Rectum and anus } & \multirow[t]{4}{*}{$\mathrm{C} 20-\mathrm{C} 21$} & 1999-2001 & 7476 & 38.6 & $37.4-39.8$ & 5733 & 41.6 & $40.2-43.0$ \\
\hline & & $2002-2004$ & 8300 & 41.4 & $40.2-42.6$ & 6328 & 45.7 & $44.3-47.0$ \\
\hline & & 2005-2007 & 9576 & 44.7 & $43.6-45.8$ & 6716 & 49.0 & $47.7-50.4$ \\
\hline & & $2008-2010$ & 9868 & 45.0 & $43.9-46.1$ & 7013 & 48.4 & $47.1-49.7$ \\
\hline \multirow[t]{4}{*}{ Colorectum } & \multirow[t]{4}{*}{$\mathrm{C} 18-\mathrm{C} 21$} & 1999-2001 & 16,647 & 40.4 & $39.5-41.2$ & 14,704 & 43.2 & $42.3-44.0$ \\
\hline & & 2002-2004 & 19,515 & 43.4 & $42.6-44.2$ & 16,921 & 47.2 & $46.4-48.1$ \\
\hline & & 2005-2007 & 23,125 & 46.7 & $45.9-47.4$ & 19,028 & 50.2 & $49.4-51.0$ \\
\hline & & 2008-2010 & 25,098 & 47.0 & $46.3-47.7$ & 20,577 & 51.0 & $50.2-51.8$ \\
\hline
\end{tabular}


Table II. Five-year relative survival rates in Poland for patients at age 15-99 with diagnosis in one of four calendar period between 1999-2010. Cont.

\begin{tabular}{|c|c|c|c|c|c|c|c|c|}
\hline \multirow[t]{2}{*}{ Site } & \multirow[t]{2}{*}{ ICD-10 } & \multirow{2}{*}{$\begin{array}{c}\text { Year of } \\
\text { diagnosis }\end{array}$} & \multicolumn{3}{|c|}{ Men } & \multicolumn{3}{|c|}{ Women } \\
\hline & & & Number & Rate & $95 \% \mathrm{Cl}$ & Number & Rate & $95 \% \mathrm{Cl}$ \\
\hline \multirow[t]{4}{*}{ Gallbladder } & $\mathrm{C} 23-\mathrm{C} 24$ & 1999-2001 & 1060 & 14.7 & $12.4-17.0$ & 3086 & 11.5 & $10.3-12.7$ \\
\hline & & $2002-2004$ & 1115 & 14.1 & $11.9-16.3$ & 3014 & 12.7 & $11.4-14.0$ \\
\hline & & $2005-2007$ & 1145 & 18.8 & $16.3-21.3$ & 2968 & 14.8 & $13.5-16.2$ \\
\hline & & $2008-2010$ & 1226 & 16.8 & $14.6-19.1$ & 3013 & 14.1 & $12.8-15.5$ \\
\hline \multirow[t]{4}{*}{ Larynx } & $\mathrm{C} 32$ & 1999-2001 & 6742 & 49.1 & $47.7-50.4$ & 815 & 58.8 & $55.1-62.4$ \\
\hline & & $2002-2004$ & 6554 & 49.3 & $47.9-50.6$ & 902 & 61.7 & $58.3-65.2$ \\
\hline & & $2005-2007$ & 6658 & 48.7 & $47.4-50.1$ & 942 & 61.3 & $57.9-64.6$ \\
\hline & & 2008-2010 & 6335 & 49.1 & $47.7-50.4$ & 891 & 58.8 & $55.3-62.3$ \\
\hline \multirow[t]{4}{*}{ Lung and trachea } & C33-C34 & 1999-2001 & 38,438 & 11.3 & $11.0-11.7$ & 10,160 & 16.8 & $16.0-17.5$ \\
\hline & & $2002-2004$ & 39,505 & 11.8 & $11.4-12.1$ & 11,750 & 17.5 & $16.8-18.2$ \\
\hline & & $2005-2007$ & 40,306 & 13.8 & $13.4-14.1$ & 13,921 & 19.3 & $18.6-20.0$ \\
\hline & & $2008-2010$ & 40,243 & 12.6 & $12.2-12.9$ & 16,226 & 18.5 & $17.9-19.2$ \\
\hline \multirow[t]{4}{*}{ Bones and cartilage } & $\mathrm{C} 40-\mathrm{C} 41$ & 1999-2001 & 531 & 40.8 & $36.3-45.2$ & 403 & 46.9 & $41.7-52.1$ \\
\hline & & 2002-2004 & 495 & 49.5 & $44.8-54.2$ & 372 & 51.4 & $45.9-56.8$ \\
\hline & & $2005-2007$ & 477 & 52.5 & $47.7-57.3$ & 385 & 57.8 & $52.6-63.1$ \\
\hline & & $2008-2010$ & 472 & 50.7 & $45.9-55.6$ & 408 & 61.0 & $55.9-66.1$ \\
\hline \multirow[t]{4}{*}{ Melanoma } & $\mathrm{C} 43$ & 1999-2001 & 2314 & 52.0 & $49.7-54.2$ & 2880 & 66.0 & $64.1-67.9$ \\
\hline & & $2002-2004$ & 2748 & 52.1 & $50.0-54.1$ & 3350 & 67.3 & $65.5-69.0$ \\
\hline & & $2005-2007$ & 3206 & 55.2 & $53.3-57.1$ & 3705 & 69.8 & $68.1-71.4$ \\
\hline & & $2008-2010$ & 3533 & 57.2 & $55.4-59.0$ & 4151 & 71.6 & $70.1-73.2$ \\
\hline \multirow[t]{4}{*}{ Breast } & C50 & 1999-2001 & & & & 34,780 & 73.0 & $72.5-73.6$ \\
\hline & & $2002-2004$ & & & & 37,173 & 75.3 & $74.8-75.8$ \\
\hline & & $2005-2007$ & & & & 42,235 & 77.0 & $76.6-77.5$ \\
\hline & & $2008-2010$ & & & & 46,633 & 78.5 & $78.1-79.0$ \\
\hline \multirow[t]{4}{*}{ Cervix } & C53 & 1999-2001 & & & & 10,367 & 53.7 & $52.7-54.7$ \\
\hline & & $2002-2004$ & & & & 10,155 & 54.2 & $53.2-55.3$ \\
\hline & & $2005-2007$ & & & & 9905 & 55.6 & $54.6-56.7$ \\
\hline & & $2008-2010$ & & & & 9486 & 56.4 & $55.4-57.5$ \\
\hline \multirow[t]{4}{*}{ Uterus } & C54 & 1999-2001 & & & & 10,624 & 74.7 & $73.8-75.6$ \\
\hline & & $2002-2004$ & & & & 12,302 & 76.0 & $75.1-76.9$ \\
\hline & & $2005-2007$ & & & & 13,680 & 76.9 & $76.1-77.8$ \\
\hline & & 2008-2010 & & & & 15,366 & 77.1 & $76.3-77.8$ \\
\hline \multirow[t]{4}{*}{ Ovary } & C56 & 1999-2001 & & & & 10,352 & 39.2 & $38.2-40.2$ \\
\hline & & $2002-2004$ & & & & 10,958 & 39.5 & $38.5-40.5$ \\
\hline & & $2005-2007$ & & & & 11,249 & 41.1 & $40.1-42.0$ \\
\hline & & 2008-2010 & & & & 11,661 & 43.9 & $42.9-44.9$ \\
\hline \multirow[t]{4}{*}{ Prostate } & C61 & 1999-2001 & 14,426 & 61.0 & $60.0-61.9$ & & & \\
\hline & & $2002-2004$ & 18,891 & 71.7 & $70.9-72.5$ & & & \\
\hline & & $2005-2007$ & 23,969 & 74.2 & $73.5-74.8$ & & & \\
\hline & & $2008-2010$ & 28,354 & 75.8 & $75.2-76.5$ & & & \\
\hline \multirow[t]{4}{*}{ Testis } & $\mathrm{C} 62$ & 1999-2001 & 1956 & 87.0 & $85.3-88.6$ & & & \\
\hline & & $2002-2004$ & 2403 & 88.2 & $86.8-89.6$ & & & \\
\hline & & $2005-2007$ & 2688 & 89.4 & $88.1-90.7$ & & & \\
\hline & & $2008-2010$ & 3238 & 91.6 & $90.5-92.6$ & & & \\
\hline
\end{tabular}


Table II. Five-year relative survival rates in Poland for patients at age 15-99 with diagnosis in one of four calendar period between 1999-2010. Cont.

\begin{tabular}{|c|c|c|c|c|c|c|c|c|}
\hline \multirow[t]{2}{*}{ Site } & \multirow[t]{2}{*}{ ICD-10 } & \multirow{2}{*}{$\begin{array}{c}\text { Year of } \\
\text { diagnosis }\end{array}$} & \multicolumn{3}{|c|}{ Men } & \multicolumn{3}{|c|}{ Women } \\
\hline & & & Number & Rate & $95 \% \mathrm{Cl}$ & Number & Rate & $95 \% \mathrm{Cl}$ \\
\hline \multirow[t]{4}{*}{ Kidney } & \multirow[t]{4}{*}{ C64-C65 } & 1999-2001 & 6206 & 51.2 & $49.8-52.6$ & 4200 & 57.4 & $55.8-59.1$ \\
\hline & & 2002-2004 & 6824 & 52.3 & $51.0-53.7$ & 4610 & 60.8 & $59.2-62.3$ \\
\hline & & 2005-2007 & 7375 & 54.7 & $53.5-56.0$ & 4975 & 63.3 & $61.8-64.8$ \\
\hline & & 2008-2010 & 8483 & 58.6 & $57.4-59.7$ & 5788 & 66.7 & $65.3-68.0$ \\
\hline \multirow[t]{4}{*}{ Urinary bladder } & \multirow[t]{4}{*}{ C67 } & 1999-2001 & 10,952 & 52.5 & $51.4-53.5$ & 2586 & 58.0 & $55.8-60.1$ \\
\hline & & 2002-2004 & 12,241 & 53.6 & $52.6-54.6$ & 3081 & 60.1 & $58.1-62.0$ \\
\hline & & 2005-2007 & 13,164 & 56.3 & $55.3-57.3$ & 3621 & 63.0 & $61.3-64.8$ \\
\hline & & 2008-2010 & 14,710 & 54.9 & $53.9-55.8$ & 4189 & 61.9 & $60.2-63.5$ \\
\hline \multirow[t]{4}{*}{ Thyroid } & \multirow[t]{4}{*}{$\mathrm{C} 73$} & 1999-2001 & 728 & 75.7 & $72.2-79.3$ & 3595 & 89.8 & $88.7-91.0$ \\
\hline & & 2002-2004 & 873 & 79.1 & $76.0-82.2$ & 4268 & 92.6 & $91.6-93.6$ \\
\hline & & 2005-2007 & 968 & 83.4 & $80.6-86.2$ & 4603 & 94.0 & $93.1-94.9$ \\
\hline & & 2008-2010 & 1086 & 84.8 & $82.2-87.4$ & 5347 & 95.1 & $94.4-95.9$ \\
\hline \multirow{4}{*}{$\begin{array}{l}\text { Hodgkin } \\
\text { lymphoma }\end{array}$} & \multirow[t]{4}{*}{ C81 } & 1999-2001 & 1228 & 69.2 & $66.4-72.1$ & 1090 & 76.4 & $73.7-79.1$ \\
\hline & & 2002-2004 & 1250 & 75.2 & $72.6-77.9$ & 1164 & 82.6 & $80.3-85.0$ \\
\hline & & 2005-2007 & 1153 & 78.8 & $76.2-81.3$ & 1156 & 82.6 & $80.2-84.9$ \\
\hline & & 2008-2010 & 1091 & 82.2 & $79.7-84.7$ & 1057 & 87.2 & $85.0-89.4$ \\
\hline \multirow{4}{*}{$\begin{array}{l}\text { Non-Hodgkin } \\
\text { lymphoma }\end{array}$} & \multirow[t]{4}{*}{$\mathrm{C} 82-\mathrm{C} 85$} & 1999-2001 & 2969 & 42.8 & $40.8-44.7$ & 2710 & 48.7 & $46.7-50.8$ \\
\hline & & $2002-2004$ & 3490 & 44.2 & $42.4-46.0$ & 2998 & 50.1 & $48.1-52.0$ \\
\hline & & 2005-2007 & 3776 & 47.9 & $46.2-49.7$ & 3439 & 54.5 & $52.7-56.3$ \\
\hline & & 2008-2010 & 4030 & 53.6 & $51.9-55.3$ & 3854 & 58.4 & $56.7-60.1$ \\
\hline \multirow[t]{4}{*}{ Multiple myeloma } & \multirow[t]{4}{*}{$\mathrm{C} 90$} & 1999-2001 & 1094 & 28.6 & $25.6-31.5$ & 1306 & 30.0 & $27.3-32.7$ \\
\hline & & 2002-2004 & 1401 & 31.2 & $28.5-33.8$ & 1624 & 32.7 & $30.3-35.2$ \\
\hline & & 2005-2007 & 1624 & 33.0 & $30.5-35.5$ & 1814 & 35.7 & $33.3-38.1$ \\
\hline & & 2008-2010 & 1722 & 38.1 & $35.5-40.6$ & 2025 & 39.8 & $37.5-42.2$ \\
\hline \multirow{4}{*}{$\begin{array}{l}\text { Lymphoid } \\
\text { leukaemia }\end{array}$} & \multirow[t]{4}{*}{ C91 } & 1999-2001 & 1691 & 46.8 & $44.2-49.5$ & 1406 & 54.0 & $51.0-56.9$ \\
\hline & & 2002-2004 & 2116 & 48.3 & $45.9-50.7$ & 1546 & 54.6 & $51.9-57.4$ \\
\hline & & 2005-2007 & 2394 & 51.9 & $49.7-54.2$ & 1857 & 60.2 & $57.7-62.7$ \\
\hline & & 2008-2010 & 2810 & 53.9 & $51.8-55.9$ & 2092 & 59.6 & $57.2-62.0$ \\
\hline \multirow[t]{4}{*}{ Myeloid leukaemia } & \multirow[t]{4}{*}{ C92 } & 1999-2001 & 1136 & 25.1 & $22.4-27.8$ & 1085 & 24.8 & $22.1-27.6$ \\
\hline & & 2002-2004 & 1293 & 25.2 & $22.7-27.8$ & 1196 & 29.7 & $26.9-32.4$ \\
\hline & & 2005-2007 & 1454 & 30.9 & $28.3-33.5$ & 1346 & 34.7 & $32.0-37.4$ \\
\hline & & 2008-2010 & 1478 & 28.1 & $25.6-30.6$ & 1366 & 34.5 & $31.8-37.2$ \\
\hline \multirow[t]{4}{*}{ All leukaemias } & \multirow[t]{4}{*}{ C91-C95 } & 1999-2001 & 3053 & 37.7 & $35.8-39.6$ & 2737 & 41.1 & $39.1-43.1$ \\
\hline & & 2002-2004 & 3649 & 39.4 & $37.6-41.1$ & 2953 & 43.4 & $41.5-45.4$ \\
\hline & & 2005-2007 & 4096 & 43.5 & $41.8-45.2$ & 3398 & 49.0 & $47.2-50.9$ \\
\hline & & 2008-2010 & 4621 & 45.0 & $43.4-46.6$ & 3803 & 49.3 & $47.6-51.1$ \\
\hline
\end{tabular}

cancer: the survival rate increased from $52.1 \%$ to $58.6 \%$ (increase by 7.4 p.p.).

Among patients with colorectal cancer (C18-C21), the survival rates increased from $40.4 \%$ to $47.9 \%$ (increase by 6.6 p.p.). The increase was observed for both, colon (C18C19), and rectal cancer (C20-C21). Survival in patients with colon cancer diagnosed in the last analyzed period was 6.5 p.p. higher as compared to patients diagnosed in the initial period included in the analysis; and for patients with rectal cancer, the respective difference was 6.4 p.p.

For patients diagnosed in the discussed decade, the increase in rate value by more than 5 p.p. was also observed 
All cancers $\mathrm{COO}-\mathrm{C97}$

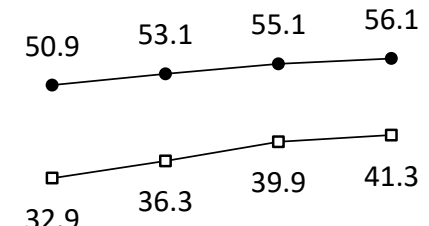

32.9

$\begin{array}{cccc} & & \square-M & \bullet-F \\ 1999 & 2002 & 2005 & 2008 \\ -2001 & -2004 & -2007 & -2010\end{array}$

Stomach C16

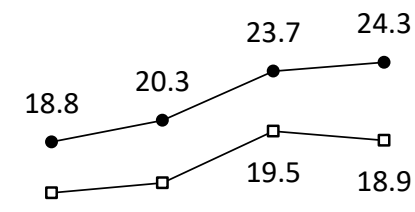

$15.2 \quad 15.9$

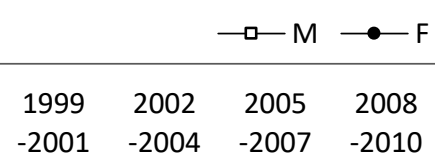

Colorectum C18-C21

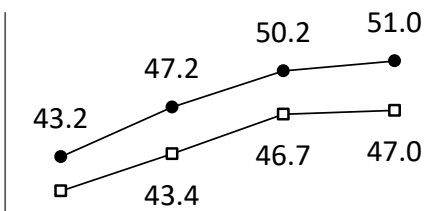

40.4

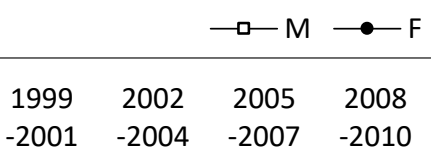

Lung C33-C34

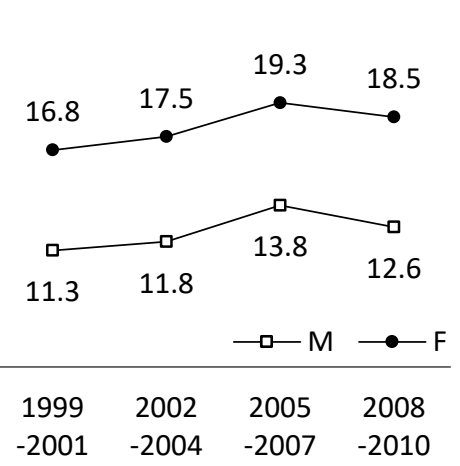

Lip, oral cavity and pharynx C00-C14

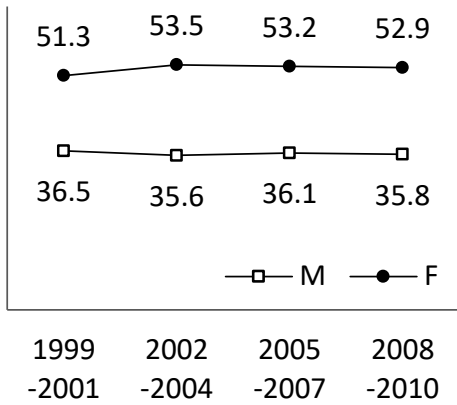

Colon C18-C19

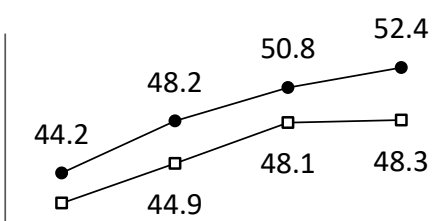

41.8

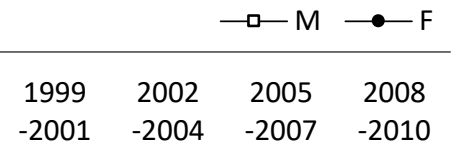

Gallbladder C23-C24

18.8

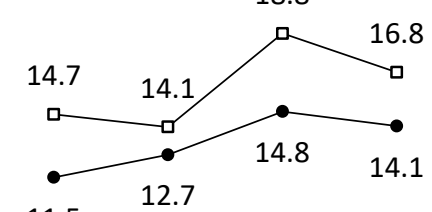

11.5

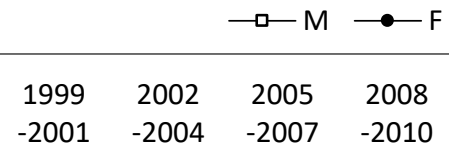

Bones and cartilage

C40-C41

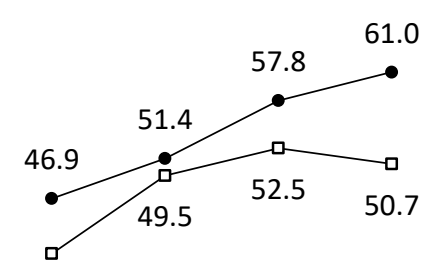

40.8

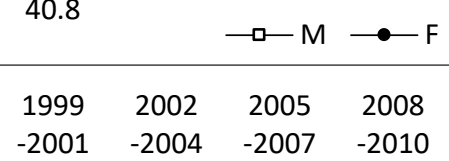

Oesophagus C15

13.2

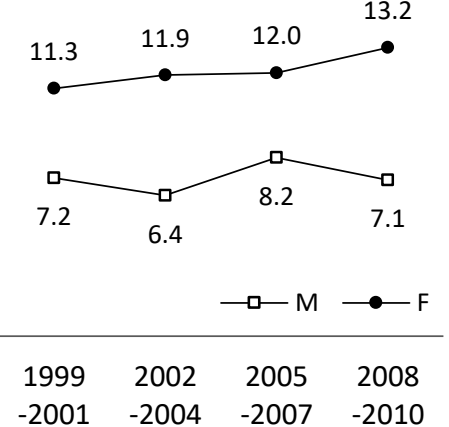

Rectum and anus C20-C21

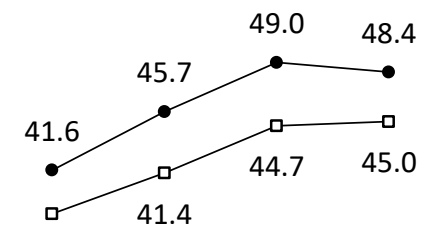

38.6

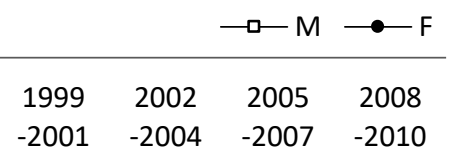

Larynx C32

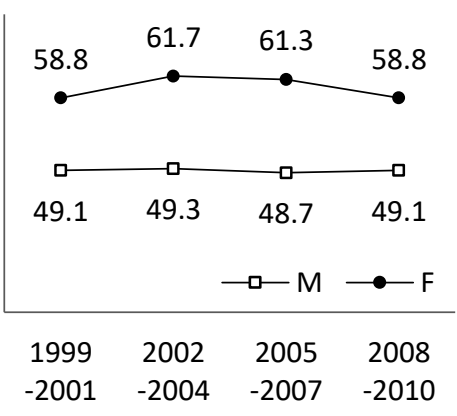

Melanoma C43

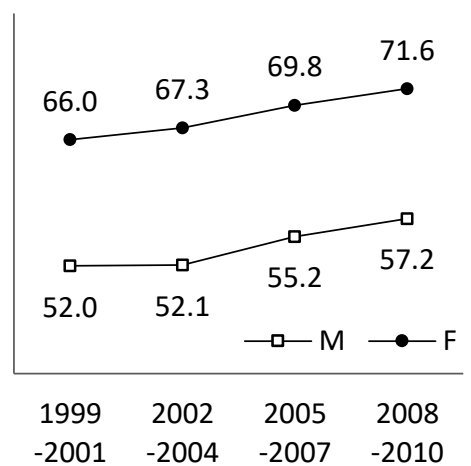

Figure 1. Trends in survival for patients at age 15-99 diagnosed in Poland during 1999-2001, 2002-2004, 2005-2007 and 2008-2010 by selected cancers 
Breast C50

Cervix C53

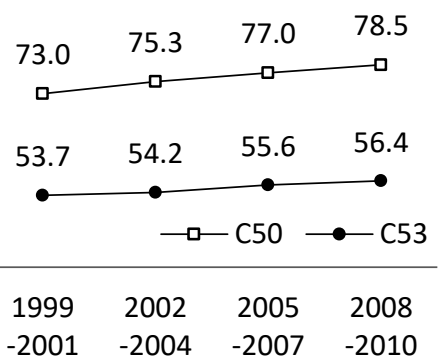

Kidney C64-C65

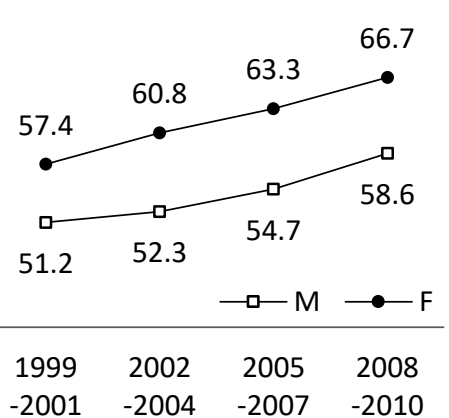

Hodgkin lymphoma C81

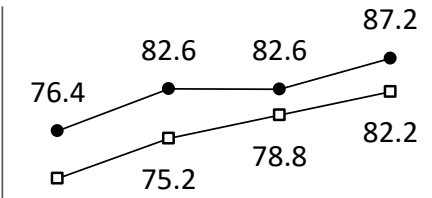

69.2

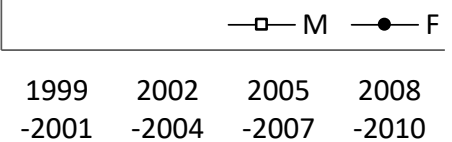

Lymphoid leukaemia C91

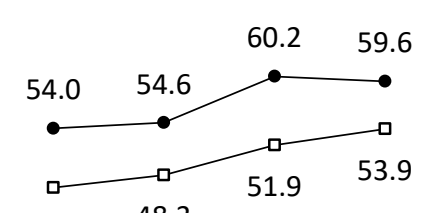

$46.8 \quad 48.3$

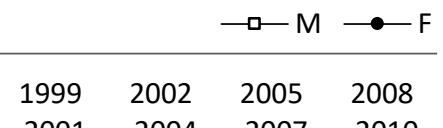

Uterus C54

Ovary C56-C57
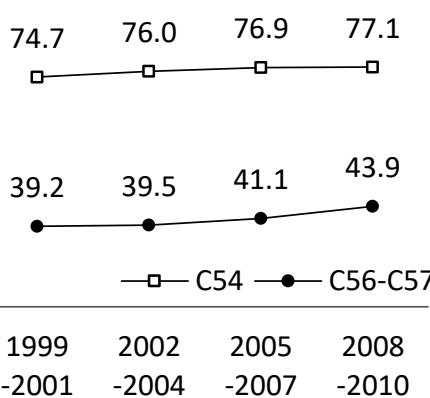

Urinary bladder C67

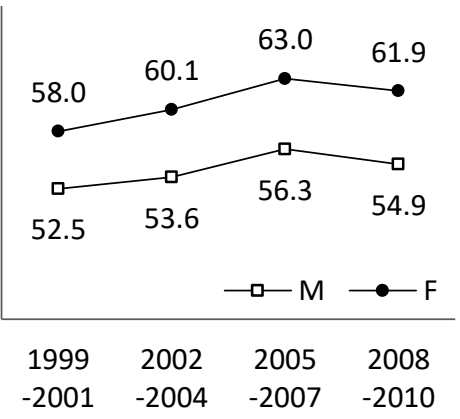

non-Hodgkin lymphomas

C82-C85+C96

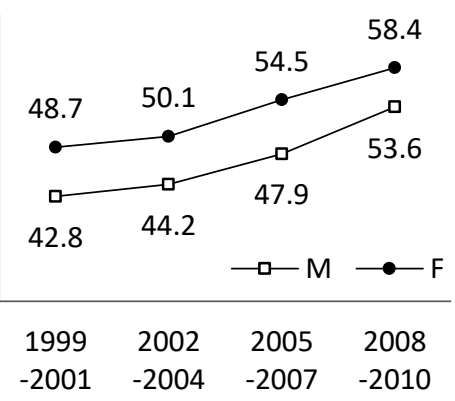

Myeloid leukaemia C92

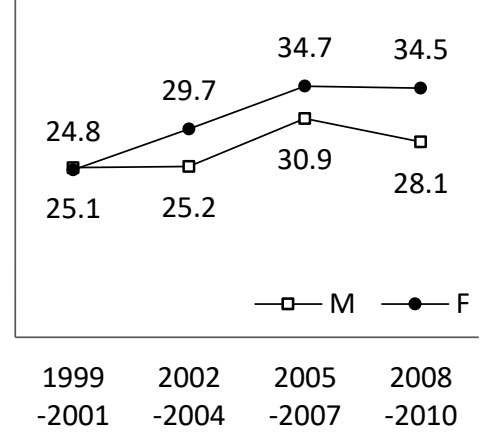

Prostate $\mathrm{C61}$

Testis C62

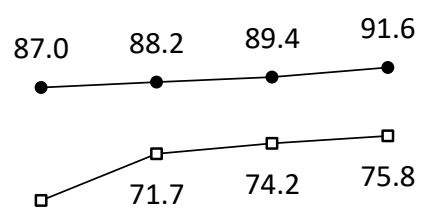

61.

$\begin{array}{cccc} & \longrightarrow & \text { C61 } & \bullet \\ & & \text { C62 } \\ 1999 & 2002 & 2005 & 2008 \\ -2001 & -2004 & -2007 & -2010\end{array}$

Thyroid C73

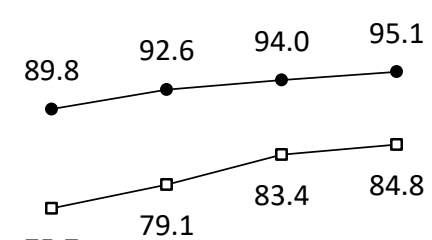

75.7

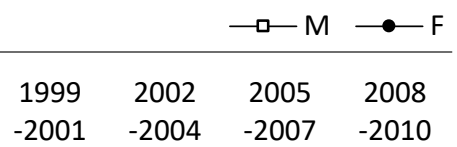

Multiple myeloma C90

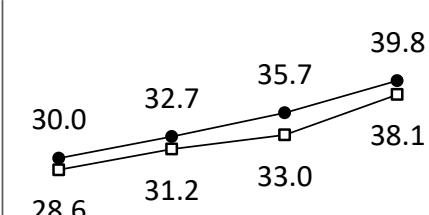

28.6

\begin{tabular}{cccc} 
& & $-\mathrm{a}$ & $\multimap$ \\
\cline { 3 - 4 } & & & \\
1999 & 2002 & 2005 & 2008 \\
-2001 & -2004 & -2007 & -2010
\end{tabular}

All leukaemias C91-C95

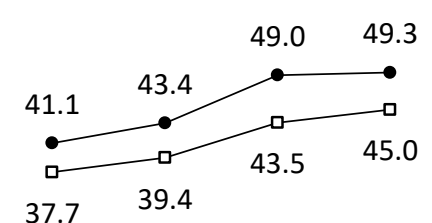

$37.7 \quad 39.4$

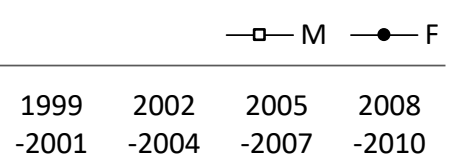

Figure 1. Trends in survival for patients at age 15-99 diagnosed in Poland during 1999-2001, 2002-2004, 2005-2007 and 2008-2010 by selected cancers. Cont. 
for melanoma cases. The improvement was also seen for patients with testicle cancer (increase from $87.0 \%$ to $91.6 \%$ — change by 4.6 p.p.).

In the case of patients with other cancers (stomach, gall bladder, lung, larynx, oesophagus cancer), the survival rate changes were minor (see Table II), and 5-year survival did not exceed $25 \%$. Among patients diagnosed over the last 3-year period, 5-year survival rates for patients with the above-mentioned cancers were as follows: stomach: $18.9 \%$, gallbladder $-16.8 \%$, lung - $12.6 \%$, oesophagus - $7.1 \%$. The lowest survival rates were observed for patients with oesophagus cancer (7.1\%) and no progress in treatment of cancer in this localization was observed. For urinary bladder and larynx cancer, the survival rates oscillated around $50 \%$ (54.9\% and $49.1 \%$, respectively), however, advance in curability of urinary bladder cancer was only 2.4 p.p., and for larynx cancer, no change was noted. No advance was found in therapy for patients with oral cavity and pharynx cancer; the survival rate remained at a similar level over the whole analysis period - at about $35 \%$.

\section{Women}

When survival rates for patients diagnosed in 1999-2010 are compared in female population (Table II, Fig. 1), the greatest improvement was observed in treatment of patients with bone and cartilage tumors (increase by 14.1 p.p. to $61 \%$ ) and hematopoietic and lymphatic tissue malignancies: Hodgkin's lymphoma (increase by 10.8 p.p., to $87.2 \%$ ), multiple myeloma (increase by 9.8 p.p., to $39.8 \%$ ), myeloid leukaemia (increase by 9.7 p.p., to $34.5 \%$ ), non-Hodgkin's lymphoma (increase by 9.7 p.p., to $58,4 \%$ ), leukaemias combined (increase by 8.3 p.p., to $49.3 \%$ ), lymphatic leukaemia (increase by 5.6 p.p., to $59.6 \%$ ). Increase in survival rates was also observed in patients with kidney cancer (increase by 9.3 p.p., to $66.7 \%$ ).

Among women, a great increase in survival rates was also observed among patients with colorectal cancer (C18C21) - increase by 7.8 p.p. (to $51.0 \%$ ), with 8.2 p.p. for colon cancer (C18-C19) (to 52.4\%) and 6.8 p.p. for rectal cancer (to 48.4\%). Improvement was obtained in survival rates for patients with melanoma (by 5.7 p.p., to $71.6 \%$ ), as well as with stomach cancer (by 5.6 p.p., to $24.3 \%$ ) and breast cancer (by 5.5 p.p., to $78.5 \%$ ).

Improvement in 5-year survival rates by slightly more than 5 p.p. was found in female patients diagnosed in 20082010 with stomach cancer ( $18.8 \%$ vs $24.3 \%$ ), breast cancer (73.0\% vs $78.5 \%$ ) and thyroid gland cancer ( $89.8 \%$ vs $95.1 \%$ ). A minor improvement over the discussed period was also noted for treatment of ovarian cancer (4.7 p.p., to $43.5 \%$ ) and urinary bladder cancer (3.9 p.p., to 61.9\%). Moreover, the survival rate improved for patients with cervical cancer by 2.8 p.p. (53.7\% vs $56.4 \%)$, with gallbladder cancer by 2.6 p.p. (11.5\% vs $14.1 \%)$, and with uterus cancer by 2.4 p.p. (74.7\% vs $77.1 \%)$. Increase in the survival rate for patients with oesophagus, lung, oral cavity and pharynx cancer was low (less than 2 p.p.). The only cancer with no change in the survival rate was larynx cancer (about 59\%).

\section{Discussion}

In the Polish National Cancer Registry, two assessments of cancer patients' survival rates for the whole Poland were performed $[13,14]$. Both analyses included patients registered in the Polish National Cancer Registry, for whom follow up was finished at the end of 2007 and 2010. Fulfilling the postulate to systematically publish the survival rates for patients from the Polish population, the authors have decided to compare four periods of diagnosis (1999-2001, 2002-2004, 2005-2007 and 2008-2010; the follow up was finished in 2006, 2009, 2012 and 2015, respectively) in order to trace changes in survival of Polish patients over the decade.

The presented population 5-year relative survival rates cover a period before and after enforcement of an act on establishing the National Cancer Control Program in 2006 [15]. Patients diagnosed in 1999-2001 were treated before the end of 2006 and enforcement of the Act did not influence their treatment results. Comparison of survival rates between the first (diagnosis in 1999-2001) and the last analyzed period (diagnosis 2008-2010) indicates that in the majority of analyzed cancers and cancers in general, significant improvement occurred (increase by 8.4 percentage points in men - from $32.9 \%$ to $41.3 \%$ and by 5 percentage points in women - from $50.9 \%$ to $56.3 \%$ ). The difference between general survival rates for men and women results from the structure of cancer incidence in the Polish population. Among cancers with favorable prognosis (relative survival rates above $50 \%$ ), only two cancer types in men (prostate with a rate of $75,8 \%$ and urinary bladder with a rate of $54.9 \%$ ) and four cancer types in women (uterus $77.1 \%$, breast $78.5 \%$, cervix $56.4 \%$, colorectal cancer $-51.0 \%$ ) are among the 5 most frequent cancers. At the same time, five most frequent cancers include lung cancer (survival rate $12.6 \%$ in men, $18.5 \%$ in women) and stomach cancer (18.9\% and $24.3 \%$, respectively), which are cancers with very unfavorable prognosis [10] (Fig. 2). Similar limitations must be considered upon comparison of cumulative survival rates for Poland and other European countries [16], since they do not account for the incidence structure in the compared countries: in men, prostate cancer prevails in the western countries (24\%), however, in Poland, lung cancer is the most frequent cancer in men (18.5\%); higher percentage of breast cancer in the incidence structure is seen in women from West European countries (30\%) than from Poland $(22 \%)[17,18]$.

Despite the use of a different measures (standardized net relative survival rate representing cumulative probability of surviving 5 or more years, assuming that cancer is the only cause of death), assessment of survival of oncology patients in Poland as compared to other countries, published within 

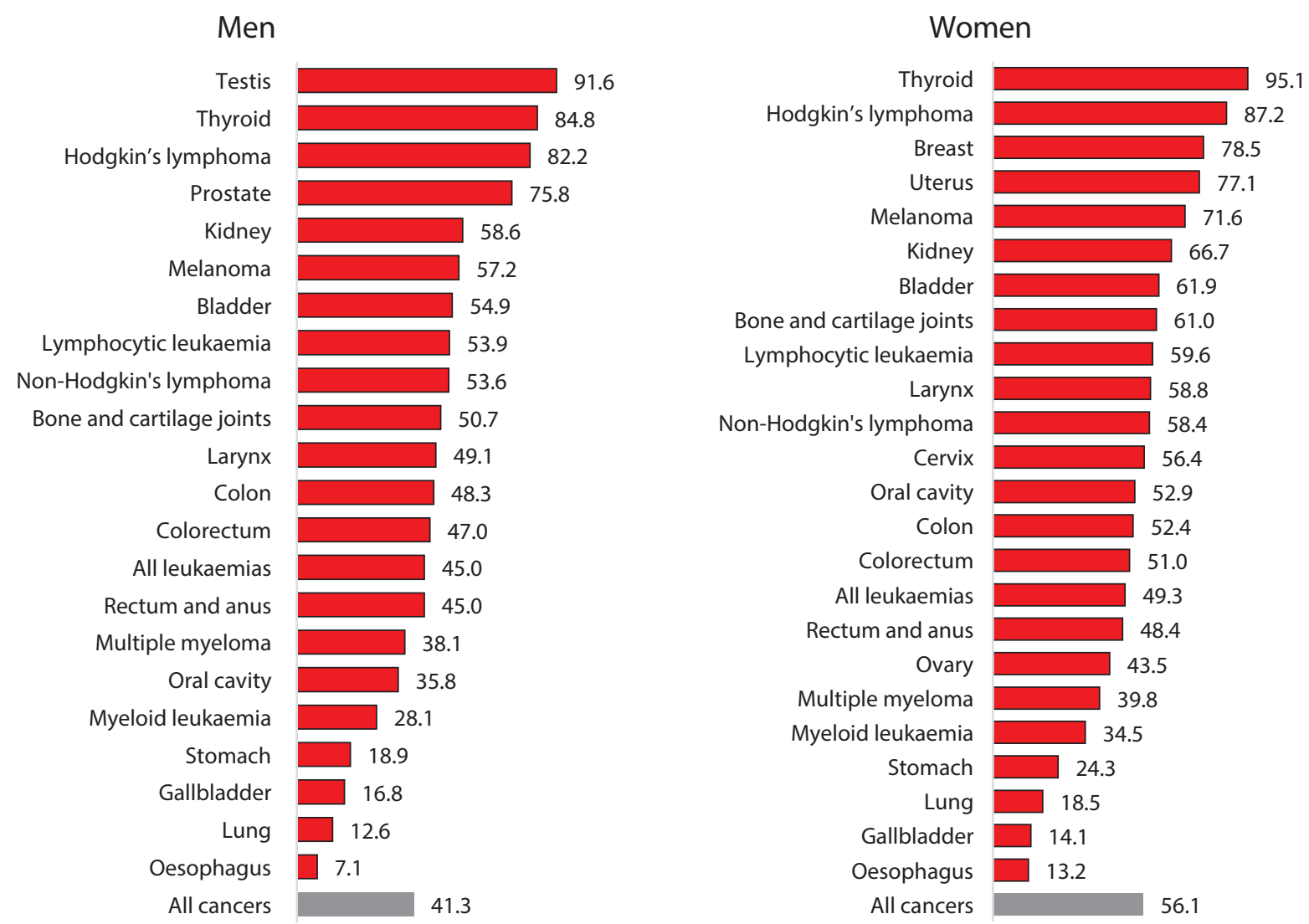

Figure 2. Trends in survival for patients at age 15-99 diagnosed in Poland during 1999-2001, 2002-2004, 2005-2007 and 2008-2010 by selected cancers

the scope of Concord-3 project [19], was consistent with results obtained in the analysis performed by the Polish National Cancer Registry. For all cancers analyzed in the Concord-3 study (15 locations in adults), net 5-year survival rates in Poland were lower than in the majority of European countries. Concord-3 study (diagnosis 2000-2014), similarly like the results presented herein (diagnosis 1999-2010), documents a major improvement that occurred in Poland in the first decade of the 21 st century.

Direct comparison of survival rates obtained using two different methods (Hakulinen method and Pohar Perme estimator) $[12,20]$ is not justified, however, comparison of tendencies observed over time indicates that improvement in treatment of cancers has taken place in Poland, regardless of the measurement method. The tendencies in the observed changes for the two studies are similar, in terms of both, direction and the magnitude of change. In Concord-3 study, in 2000-2014, increase in net survival rate by about 6-8 p.p. for colorectal cancer (both sexes) was shown (increase from $45.3 \%$ to $52,9 \%$ for colon cancer and from $42.5 \%$ to $48.4 \%$ for rectal cancer), whereas in the PNCR analysis, the increase between the first and the last analyzed period (1999-2001 vs 2008-2010) was, respectively, 6.5 p.p. and 6.4 p.p. for men, and 8.2 p.p. and 6.8 p.p. for women. For the other analyzed cancers, improvement of survival was also found: stomach cancer - according to Concord-3, increase by 5 p.p. (from $15.9 \%$ to $19.9 \%$ ), according to $P N C R$, increase by 3.7 p.p. in men and by 5.5 p.p. in women; lung cancer - according to Concord-3, increase by 2.3 p.p. (from $12.1 \%$ to $14.4 \%$ ), according to $P N C R$, increase by 1.3 p.p. in men and 1.8 p.p. in women; breast cancer - according to Concord-3, increase by 5.2 p.p. (from $71.3 \%$ to $76.5 \%$ ), according to PNCR, increase by 5.5 p.p.; cervical cancer - according to Concord-3, increase by 3.5 p.p. (from $51.6 \%$ to $55.1 \%$ ), according to PNCR, increase by 2.8 p.p.; ovarian cancer - according to Concord-3, increase by 4.8 p.p. (from $32.7 \%$ to $37.5 \%$ ), according to PNCR, 4.7 p.p.

In the case of prostate cancer patients, in Concord-3 study, the survival rates increased by 9.3 p.p. (68.8 vs $78.1 \%$ ) whereas in PNCR analysis, the increase was estimated as 14.9 p.p. Such a significant increase results probably from more and more common opportunistic screening for prostate cancer (assaying PSA level and using TRUS examination for diagnostic purposes). According to Prajsner et al. who analyzed the results of PolSenior study performed in 2007-2012 [21], $41.2 \%$ of older men (aged $65-74$ ) and $24.8 \%$ of younger men (aged 55-59) had PSA level assayed. Early beginning of prostate cancer diagnostics may lead to detection of early 
non-symptomatic cancer, which in turn may result in the so-called overtreatment, and to fast change in the value of survival rates by detection of the disease before manifestation of clinical symptoms (the so-called lead time bias). In 2000 in the U.S., Medicare started funding an annual PSA examination, which resulted in rapid increase in incidence and had no effect on mortality [22].

The greatest improvement occurred for treatment of relatively rare cancers in Poland. 5-year relative survival rates for patients with Hodgkin's lymphoma, non-Hodgkin's lymphoma and with bone and cartilage malignancies increased in the analyzed years by more than 10 percentage points. Improvement was also obtained in treatment of patients with myeloma and some leukaemia types.

The presented results are a significant generalization and may not be referred to individual clinical situations. The discussed analysis is a population-based assessment of oncological care in Poland. Over the last two decades, a significant improvement in diagnostics and therapy of cancers occurred globally, hence improving both prognosis and patient survival. In Poland, high cost of modern therapies is still a big problem, which significantly limits and delays their common use. Although the survival rates are lower than in other countries, the increase in their values observed in the first decade of the 21 st century may result from activities within the scope of the National Cancer Control Program enforced in 2006.

\section{Conclusions}

1. Over the discussed period, significant improvement in survival for the majority of cancers in Poland occurred, although survival rates for Poland are still lower than those for other European countries.

2. The presented results are consistent with results obtained within the scope of the international CONCORD-3 project (in terms of both, rate values and tendency over time).

3. Especially high increase in survival rates for patients with prostate cancer should be related to growing usage of PSA testing and TRUS examination and hence with detection of early forms of this cancer.

4. The highest increase in the survival rate values was observed for rare cancers (Hodgkin's lymphoma, non-Hodgkin's lymphoma, bone and cartilage malignancies and some leukaemias).

5. It seems that the National Cancer Control Program (2006-2015) influenced improvement in efficiency of treatment of malignant neoplasms in Poland.

Conflict of interest: none declared

\section{Urszula Wojciechowska, MD, PhD}

The Maria Skłodowska-Curie Memorial Cancer Center and Institute of Oncology
Department of Epidemiology

ul. Roentgena 5, 02-781 Warszawa, Poland

e-mail: urszula.wojciechowska@coi.pl

Received: 30 Nov 2017

Accepted: 13 Feb 2018

\section{References}

1. World Cancer Report 2014. Stewart BW, Wild CP (ed.). Lyon: International Agency for Research on Cancer, 2014.

2. Waligórska M, Kostrzewa Z, Potyra M et al. Prognoza ludności na lata 2014-2050. Warszawa: GUS, Studia i Analizy, 2014

3. http://12sposobownazdrowie.pl/.

4. Pinsky PF. Principles of cancer screening. Surg Clin North Am 2015; 95: 953-966.

5. Forrest LF, Adams J, Wareham $\mathrm{H}$ et al. Socioeconomic inequalities in lung cancer treatment: systematic review and meta-analysis. PLoS Med 2013; 10: e1001376.

6. Sunkara V, Herbert JR. The colorectal cancer mortality-to-incidence ratio as a potential cancer surveillance measure in Asia. Asian Pac J Cancer Prev 2016; 17: 4323-4326.

7. Choi $E$, Lee $S$, Nhung $B C$ et al. Cancer mortality-to-incidence ratio as an indicator of cancer management outcomes in Organization for Economic Cooperation and Development countries. Epidemiol Health 2017; 39: e2017006. doi: 10.4178/epih.e2017006.

8. Pesmen C. What five years really means. Survival statistics mean different things for different cancers (March 16, 2007). https://www. curetoday.com/publications/cure/2007/spring2007/what-five-yearsreally-means. Accessed on 15 November 2017.

9. Cancer registration: Principles and methods. Jensen OM, Parkin DM, MacLennan R et al. (ed.). Lyon: IARC Scientific Publications No 95, 1995.

10. Wojciechowska U, Olasek P, Czauderna K et al. Nowotwory złośliwe w Polsce w 2014 roku. Warszawa: Centrum Onkologii — Instytut, 2016.

11. ICD-10: International Statistical Classification of Diseases and Related Health Problems. Volume I. Geneva: WHO, 2010.

12. Hakulinen T. Cancer survival corrected for heterogeneity in patient withdrawal. Biometrics 1982; 38: 933-942.

13. Wojciechowska U, Didkowska J, Zatoński W. Pięcioletnie przeżycia chorych na nowotwory w Polsce. Nowotwory J Oncol 2010; 60: 122-128.

14. Wojciechowska U, Didkowska J. Poprawa przeżyć chorych na nowotwory złośliwe w Polsce. Analiza przeżyć pacjentów zdiagnozowanych w latach 2003-2005. Nowotwory J Oncol 2013; 63: 279-285. doi: $10.5603 /$ NJO.2013.0014.

15. The act of 1 July 2005 on establishing a many-year program entitled "The National Cancer Control Program". Dz.U. (Polish Journal of Law) of 2005 , No. 143 , Pos. 1200.

16. Rachtan J. Jakie informacje przynosi badanie EUROCARE-4. Medycyna Paliatywna - Onkologia 2009; 5: 9.

17. Ferlay J, Soerjomataram I, Ervik M et al. (ed.) GLOBOCAN 2012 v1.0: Cancer Incidence and Mortality Worldwide: IARC CancerBase No. 11 [Internet]. Lyon: International Agency for Research on Cancer, 2013. http://globocan.iarc.fr, accessed on 15.10.2017.

18. Wojciechowska U, Didkowska J. Zachorowania i zgony na nowotwory złośliwe w Polsce. Warszawa: Krajowy Rejestr Nowotworów, Centrum Onkologii - Instytut im. Marii Skłodowskiej-Curie. Access at http:// onkologia.org.pl/raporty/,15.10.2017.

19. Allemani $\mathrm{C}$, Weir HK, Carreira $\mathrm{H}$ et al. Global surveillance of cancer survival 1995-2009: analysis of individual data for 25,676,887 patients from 279 population-based registries in 67 countries (CONCORD-2). Lancet 2015; 385: 977-1010.

20. Perme MP, Henderson R, Stare J. An approach to estimation in relative survival regression. Biostatistics 2009; 10: 136-146.

21. Prajsner A, Chudek J, Szybalska A et al. PolSenior Study Group. Socioeconomic determinants of prostate-specific antigen testing and estimation of the prevalence of undiagnosed prostate cancer in an elderly Polish population based on the PolSenior study. Arch Med Sci 2016; 12: 1028-1035. doi: 10.5114/aoms.2015.55271.

22. Shao $\mathrm{YH}$, Albertsen $\mathrm{PC}$, Shih $\mathrm{W}$ et al. The impact of prostate specific antigen testing frequency on prostate cancer incidence and treatment in older men. Prostate Cancer Prostatic Dis 2011; 14: 332-339. 